Tohoku Math. J.

54 (2002), 163-177

\title{
THE BEHAVIOR OF THE PRINCIPAL DISTRIBUTIONS ON THE GRAPH OF A HOMOGENEOUS POLYNOMIAL
}

\author{
NAOYA ANDO
}

(Received April 28, 2000, revised February 5, 2001)

\begin{abstract}
In this paper, we shall study the behavior of the principal distributions on the graph of a homogeneous polynomial in two variables such that the set of its umbilical points is finite. In particular, we shall present a method of describing the indices of the umbilical points and the point at infinity.
\end{abstract}

1. Introduction. Let $S$ be a smooth surface in $\boldsymbol{R}^{3}$ and $\operatorname{Umb}(S)$ the set of the umbilical points of $S$, and set $\operatorname{Reg}(S):=S \backslash \operatorname{Umb}(S)$. If $\operatorname{Reg}(S) \neq \varnothing$, then there exists a principal distribution $\mathrm{D}_{S}$ on $S$, which is a one-dimensional continuous distribution on $\operatorname{Reg}(S)$ such that $\mathrm{D}_{S}(p)$ is a principal direction at $p \in \operatorname{Reg}(S)$. The behavior of $\mathrm{D}_{S}$ around a non-umbilical point $p \in \operatorname{Reg}(S)$ is easily described. Namely, it is represented by a vector field which is nonzero at $p$. On the other hand, the behavior around an umbilical point $p_{0} \in \operatorname{Umb}(S)$ may be very complicated. Generally, it is not always represented by any vector field. Let $p_{0}$ be isolated as an umbilical point. Then as a quantity in relation to the behavior of $\mathrm{D}_{S}$ around $p_{0}$, the index $\operatorname{ind}_{p_{0}}(S)$ of $p_{0}$ is defined ([5, pp. 137]).

Let $P_{F}^{k}$ denote the set of the homogeneous polynomials of degree $k \geqq 3$ in two variables such that the set of the umbilical points on each of their graphs is finite. Let $f$ be an element of $P_{F}^{k}$ and $\mathrm{G}_{f}$ the graph of $f$, and $\tilde{\mathrm{G}}_{f}$ denote the topological space obtained by the onepoint compactification for $\mathbf{G}_{f}$. Denote by $\infty$ the point added to $\mathbf{G}_{f}$, and $\operatorname{set} \operatorname{Sing}\left(\tilde{\mathbf{G}}_{f}\right):=$ $\operatorname{Umb}\left(\mathrm{G}_{f}\right) \cup\{\infty\}$. Inducing the natural differentiable structure on $\tilde{\mathrm{G}}_{f}$, one may consider any principal distribution on $\mathbf{G}_{f}$ as a distribution on $\tilde{\mathbf{G}}_{f} \backslash \operatorname{Sing}\left(\tilde{\mathbf{G}}_{f}\right)$. The purpose of this paper is to present a method of describing the index $\operatorname{ind}_{p_{0}}\left(\tilde{\mathbf{G}}_{f}\right)$ of each $p_{0} \in \operatorname{Sing}\left(\tilde{\mathbf{G}}_{f}\right)$.

Let $o$ denote the origin of $\boldsymbol{R}^{3}$. Let $r_{o}, r_{\infty}$ be positive numbers satisfying

$$
\mathrm{Umb}\left(\mathrm{G}_{f}\right) \backslash\left\{r_{o}^{2}<x^{2}+y^{2}<r_{\infty}^{2}\right\}=\{o\},
$$

and $\mathrm{D}_{f}^{(1)}, \mathrm{D}_{f}^{(2)}$ two principal distributions on $\mathrm{G}_{f}$ which give the principal directions at each point of $\operatorname{Reg}\left(\mathrm{G}_{f}\right)$. For $i=1,2$ and for $\omega=o, \infty$, let $\phi_{r_{\omega}}^{(i)}$ be a continuous function on $\boldsymbol{R}$ satisfying

$$
\cos \phi_{r_{\omega}}^{(i)}(\theta) \frac{\partial}{\partial x}+\sin \phi_{r_{\omega}}^{(i)}(\theta) \frac{\partial}{\partial y} \in \mathrm{D}_{f}^{(i)}\left(r_{\omega} \cos \theta, r_{\omega} \sin \theta\right)
$$

for any $\theta \in \boldsymbol{R}$. In Section 3, we shall prove the following:

Proposition 1.1. For $i \in\{1,2\}, \omega, \omega_{1}, \omega_{2} \in\{o, \infty\}$ and for $\theta_{0} \in \boldsymbol{R}$, 
(1) there exists a continuous function $\phi_{\omega, \theta_{0}}^{(i)}$ on $(0, \infty)$ satisfying

(a) $\phi_{\omega, \theta_{0}}^{(i)}\left(r_{\omega}\right)=\phi_{r_{\omega}}^{(i)}\left(\theta_{0}\right)$, and

(b) for any $\rho \in(0, \infty), \cos \phi_{\omega, \theta_{0}}^{(i)}(\rho) \partial / \partial x+\sin \phi_{\omega, \theta_{0}}^{(i)}(\rho) \partial / \partial y$ is in a principal direction at $\left(\rho \cos \theta_{0}, \rho \sin \theta_{0}\right)$;

(2) there exist numbers $\phi_{\omega, o}^{(i)}\left(\theta_{0}\right), \phi_{\omega, \infty}^{(i)}\left(\theta_{0}\right)$ satisfying

$$
\lim _{\rho \rightarrow 0} \phi_{\omega, \theta_{0}}^{(i)}(\rho)=\phi_{\omega, o}^{(i)}\left(\theta_{0}\right), \quad \lim _{\rho \rightarrow \infty} \phi_{\omega, \theta_{0}}^{(i)}(\rho)=\phi_{\omega, \infty}^{(i)}\left(\theta_{0}\right)
$$

(3) there exist numbers $\phi_{\omega_{1}, \omega_{2}}^{(i)}\left(\theta_{0}+0\right), \phi_{\omega_{1}, \omega_{2}}^{(i)}\left(\theta_{0}-0\right)$ satisfying

$$
\lim _{\theta \rightarrow \theta_{0} \pm 0} \phi_{\omega_{1}, \omega_{2}}^{(i)}(\theta)=\phi_{\omega_{1}, \omega_{2}}^{(i)}\left(\theta_{0} \pm 0\right) \text {. }
$$

We set

$$
\begin{aligned}
& \Gamma_{\omega_{1}, \omega_{2}}^{(i)}\left(\theta_{0}\right):=\phi_{\omega_{1}, \omega_{2}}^{(i)}\left(\theta_{0}+0\right)-\phi_{\omega_{1}, \omega_{2}}^{(i)}\left(\theta_{0}-0\right), \\
& S_{\omega}:=\left\{\theta_{0} \in \boldsymbol{R} ; \prod_{i=1}^{2} \Gamma_{\omega, \omega}^{(i)}\left(\theta_{0}\right) \neq 0\right\}, \\
& S_{u}:=\left\{\theta_{0} \in \boldsymbol{R} ; \prod_{\left\{\omega_{1}, \omega_{2}\right\}=\{o, \infty\}} \prod_{i=1}^{2} \Gamma_{\omega_{1}, \omega_{2}}^{(i)}\left(\theta_{0}\right) \neq 0\right\} .
\end{aligned}
$$

For an integer $n \in \boldsymbol{Z}$, let $I_{n}$ be the subset of $\boldsymbol{R}$ defined by

$$
I_{n}:= \begin{cases}\{n \pi / 2\} & \text { if } n \text { is even } \\ ((n-1) \pi / 2,(n+1) \pi / 2) & \text { if } n \text { is odd }\end{cases}
$$

In Section 4, we shall prove the following:

Proposition 1.2. For $\omega \in\{o, \infty, u\}$, the following hold:

(1) the set $S_{\omega} \cap[\theta, \theta+\pi)$ for $\theta \in \boldsymbol{R}$ is finite and the number $\sharp\left\{S_{\omega} \cap[\theta, \theta+\pi)\right\}$ does not depend on $\theta$;

(2) For $\theta_{0} \in S_{o} \cup S_{\infty} \cup S_{u}$ and for $\omega_{1}, \omega_{2} \in\{o, \infty\}$, there exists an integer $v_{\omega_{1}, \omega_{2}}\left(\theta_{0}\right)$ satisfying $\Gamma_{\omega_{1}, \omega_{2}}^{(i)}\left(\theta_{0}\right) \in I_{\nu_{\omega_{1}, \omega_{2}}\left(\theta_{0}\right)}$ for $i=1,2$.

For $\theta \in \boldsymbol{R}$, let $\operatorname{Hess}_{f}(\theta)$ be the Hessian of $f$ at $(\cos \theta, \sin \theta)$, and $\eta$ a continuous function on $\boldsymbol{R}$ such that ${ }^{t}(\cos \eta(\theta), \sin \eta(\theta))$ is an eigenvector of Hess $f(\theta)$ for any $\theta \in \boldsymbol{R}$. We set $\tilde{f}(\theta):=f(\cos \theta, \sin \theta)$. In addition, we set

$$
\begin{aligned}
& Z_{f}:=\left\{\theta_{0} \in \boldsymbol{R} ; \tilde{f}\left(\theta_{0}\right)=0\right\}, \\
& Z_{f}^{\prime}:=\left\{\theta_{0} \in Z_{f} ; \frac{d \tilde{f}}{d \theta}\left(\theta_{0}\right) \neq 0\right\}, \quad Z_{f}^{\prime \prime}:=\left\{\theta_{0} \in Z_{f} ; \frac{d \tilde{f}}{d \theta}\left(\theta_{0}\right)=0\right\} .
\end{aligned}
$$

The main theorem in this paper is the following:

THEOREM 1.3. Let $f$ be an element of $P_{F}^{k}$. Then

(1) (a) $\theta_{0} \in S_{o}$ holds if and only if $\operatorname{Hess}_{f}\left(\theta_{0}\right)$ is a scalar matrix,

(b) $\theta_{0} \in S_{o}$ satisfies $v_{o, o}\left(\theta_{0}\right)=-1$, and 
(c) for any $\theta \in \boldsymbol{R}$, the following holds:

$$
\operatorname{ind}_{o}\left(\tilde{\mathbf{G}}_{f}\right)=\frac{\eta(\theta+2 \pi)-\eta(\theta)}{2 \pi}-\frac{\sharp\left\{S_{o} \cap[\theta, \theta+\pi)\right\}}{2} ;
$$

(2) (a) $\theta_{0} \in S_{\infty}$ satisfies $\operatorname{det}\left(\operatorname{Hess}_{f}\left(\theta_{0}\right)\right)=0$ and $v_{\infty, \infty}\left(\theta_{0}\right) \in\{1,-1,-2\}$, and

(b) for any $\theta \in \boldsymbol{R}$, the following holds:

$$
\begin{aligned}
\operatorname{ind}_{\infty}\left(\tilde{\mathbf{G}}_{f}\right)=1 & +\frac{1}{2} \sharp\left\{Z_{f}^{\prime} \cap[\theta, \theta+\pi)\right\}+\sharp\left\{Z_{f}^{\prime \prime} \cap[\theta, \theta+\pi)\right\} \\
& -\frac{1}{2} \sum_{\theta_{0} \in S_{\infty} \cap[\theta, \theta+\pi)} v_{\infty, \infty}\left(\theta_{0}\right) ;
\end{aligned}
$$

(3) (a) $\theta_{0} \in S_{u}$ holds if and only if on $\left\{\left(\rho \cos \theta_{0}, \rho \sin \theta_{0}\right)\right\}_{\rho>0}$, there exists an umbilical point $p\left(\theta_{0}\right)$ satisfying $\operatorname{ind}_{p\left(\theta_{0}\right)}\left(\tilde{\mathrm{G}}_{f}\right) \neq 0$, and

(b) $\theta_{0} \in S_{u}$ satisfies

$$
\left(v_{\infty, o}\left(\theta_{0}\right), v_{o, \infty}\left(\theta_{0}\right), \operatorname{ind}_{p\left(\theta_{0}\right)}\left(\tilde{\mathbf{G}}_{f}\right)\right) \in\{(2,-2,1 / 2),(-2,2,-1 / 2)\} .
$$

We shall prove (1) (resp. (2), (3)) of Theorem 1.3 in Section 5 (resp. Section 6, Section 7).

In our previous paper [1], we studied the behavior of the principal distributions on $\mathbf{G}_{f}$ around $o$, and showed that ind ${ }_{o}\left(\mathrm{G}_{f}\right) \in\{1+i-k / 2\}_{i=0}^{[k / 2]}$. We have further studied the behavior of the principal distributions around $o$, in relation to the existence of other umbilical points of $\mathbf{G}_{f}$ than $o$ and the behavior of the gradient vector field of $f$ ([2]).

It is known that if $S$ is a surface with constant mean curvature, then an umbilical point which is not contained in the interior of $\operatorname{Umb}(S)$ is isolated and its index is negative ([5, pp. 139]). More generally, if $S$ is a special Weingarten surface, then the same result is obtained ([4]).

It has been expected that for any smooth surface $S$ with an isolated umbilical point $p_{0}$, $\operatorname{ind}_{p_{0}}(S) \leqq 1$ holds. If this conjecture is affirmatively solved, then Hopf-Poincaré's theorem implies that the number of the umbilical points on a compact, orientable surface of genus 0 is more than or equal to two, and this immediately gives the affirmative answer to Carathéodory's conjecture on the number of the umbilical points on a compact, strictly convex surface.

Let $F$ be a smooth, real-valued function of two real variables and set $\partial_{\bar{z}}:=(\partial / \partial x+$ $\sqrt{-1} \partial / \partial y) / 2$. Then Loewner's conjecture for a natural number $n \in N$ says that if the vector field $\operatorname{Re}\left(\partial_{\bar{z}}^{n} F\right) \partial / \partial x+\operatorname{Im}\left(\partial_{\bar{z}}^{n} F\right) \partial / \partial y$ has an isolated zero point $z_{0}$, then its index is less than or equal to $n$ ([9], [6]). It is known that Loewner's conjecture for $n=2$ is equivalent to the above conjecture that ind $p_{0}(S) \leqq 1$ ([8]). As for recent papers in relation to Carathéodory's and Loewner's conjectures, [3], [7], [8] may be found.

The author is a research fellow of the Japan Society for the Promotion of Science. The author is grateful to Professor T. Ochiai for his helpful advice and constant encouragement.

2. A gradient root. Let $P_{o}^{k}$ denote the set of the homogeneous polynomials of degree $k \geqq 3$ in two variables such that on each of their graphs, $o$ is an isolated umbilical point. For 
$f \in P_{o}^{k}$, we set

$$
p_{f}:=\frac{\partial f}{\partial x}, \quad q_{f}:=\frac{\partial f}{\partial y}, \quad r_{f}:=\frac{\partial^{2} f}{\partial x^{2}}, \quad s_{f}:=\frac{\partial^{2} f}{\partial x \partial y}, \quad t_{f}:=\frac{\partial^{2} f}{\partial y^{2}} .
$$

Moreover, we define

$$
\begin{aligned}
\tilde{p}_{f}(\theta):=p_{f}(\cos \theta, \sin \theta), & \tilde{q}_{f}(\theta):=q_{f}(\cos \theta, \sin \theta), \\
\tilde{r}_{f}(\theta):=r_{f}(\cos \theta, \sin \theta), & \tilde{s}_{f}(\theta):=s_{f}(\cos \theta, \sin \theta), \\
\tilde{t}_{f}(\theta):=t_{f}(\cos \theta, \sin \theta), &
\end{aligned}
$$

and

$$
\begin{aligned}
d_{f}(\theta, \phi):= & \tilde{s}_{f}(\theta) \cos ^{2} \phi+\left\{\tilde{t}_{f}(\theta)-\tilde{r}_{f}(\theta)\right\} \cos \phi \sin \phi-\tilde{s}_{f}(\theta) \sin ^{2} \phi, \\
n_{f}(\theta, \phi):= & \left\{\tilde{s}_{f}(\theta) \tilde{p}_{f}(\theta)^{2}-\tilde{p}_{f}(\theta) \tilde{q}_{f}(\theta) \tilde{r}_{f}(\theta)\right\} \cos ^{2} \phi \\
& +\left\{\tilde{t}_{f}(\theta) \tilde{p}_{f}(\theta)^{2}-\tilde{r}_{f}(\theta) \tilde{q}_{f}(\theta)^{2}\right\} \cos \phi \sin \phi \\
& +\left\{\tilde{p}_{f}(\theta) \tilde{q}_{f}(\theta) \tilde{t}_{f}(\theta)-\tilde{s}_{f}(\theta) \tilde{q}_{f}(\theta)^{2}\right\} \sin ^{2} \phi .
\end{aligned}
$$

Then $\left(\rho_{0}, \theta_{0}, \phi_{0}\right)$ satisfies the equation

$$
\rho_{0}^{k-2} d_{f}\left(\theta_{0}, \phi_{0}\right)+\rho_{0}^{3 k-4} n_{f}\left(\theta_{0}, \phi_{0}\right)=0
$$

if and only if a tangent vector $\cos \phi_{0} \partial / \partial x+\sin \phi_{0} \partial / \partial y$ at $\left(\rho_{0} \cos \theta_{0}, \rho_{0} \sin \theta_{0}\right)$ is in a principal direction ([1]). We set

$$
\operatorname{grad}_{f}(\theta):=\left(\begin{array}{l}
\tilde{p}_{f}(\theta) \\
\tilde{q}_{f}(\theta)
\end{array}\right), \quad \operatorname{Hess}_{f}(\theta):=\left(\begin{array}{cc}
\tilde{r}_{f}(\theta) & \tilde{s}_{f}(\theta) \\
\tilde{s}_{f}(\theta) & \tilde{t}_{f}(\theta)
\end{array}\right), \quad u_{\phi}:=\left(\begin{array}{c}
\cos \phi \\
\sin \phi
\end{array}\right) .
$$

We denote by $\langle$,$\rangle the scalar product in \boldsymbol{R}^{2}$. Then, since

$$
\operatorname{grad}_{f}(\theta)=\frac{1}{k-1} \operatorname{Hess}_{f}(\theta) u_{\theta},
$$

we obtain

LEMMA 2.1. The following hold:

$$
\begin{aligned}
& d_{f}(\theta, \phi)=\left\langle\operatorname{Hess}_{f}(\theta) u_{\phi}, u_{\phi+\pi / 2}\right\rangle, \\
& n_{f}(\theta, \phi)=c_{k}(\theta)\left\langle\operatorname{Hess}_{f}(\theta) u_{\theta}, u_{\phi}\right\rangle \sin (\phi-\theta),
\end{aligned}
$$

where $c_{k}(\theta):=\operatorname{det}\left(\operatorname{Hess}_{f}(\theta)\right) /(k-1)^{2}$.

A number $\theta_{0}$ is said to be $a$ gradient root of $f$ if $(d \tilde{f} / d \theta)\left(\theta_{0}\right) \operatorname{det}\left(\operatorname{Hess}_{f}\left(\theta_{0}\right)\right)=0$ holds. The set of the gradient roots of $f$ is denoted by $R_{f}^{G}$.

PROPOSITION 2.2. For $\theta_{0} \in \boldsymbol{R}, \theta_{0} \in R_{f}^{G}$ holds if and only if there exists a number $\xi \in[0, \pi)$ such that for any $\phi \in \boldsymbol{R}$, the following holds:

$$
(\cos \xi) d_{f}\left(\theta_{0}, \phi\right)+(\sin \xi) n_{f}\left(\theta_{0}, \phi\right)=0 .
$$

Moreover, such a number $\xi \in[0, \pi)$ is uniquely determined by $\theta_{0} \in R_{f}^{G}$. 
Proof. Suppose $\operatorname{det}\left(\operatorname{Hess}_{f}\left(\theta_{0}\right)\right)=0$. Then we obtain $n_{f}\left(\theta_{0}, \phi\right)=0$ for any $\phi \in \boldsymbol{R}$. Since $f \in P_{o}^{k}$, we see that $\operatorname{Hess}_{f}\left(\theta_{0}\right)$ is nonzero, and $d_{f}\left(\theta_{0}, \phi\right) \neq 0$ holds for some $\phi \in \boldsymbol{R}$. Suppose $\operatorname{det}\left(\operatorname{Hess}_{f}\left(\theta_{0}\right)\right) \neq 0$ and $(d \tilde{f} / d \theta)\left(\theta_{0}\right)=0$. Then by (2.2) together with

$$
\frac{d \tilde{f}}{d \theta}(\theta)=\left\langle\operatorname{grad}_{f}(\theta), u_{\theta+\pi / 2}\right\rangle,
$$

we find numbers $d\left(\theta_{0}\right) \in \boldsymbol{R}$ and $n\left(\theta_{0}\right) \in \boldsymbol{R} \backslash\{0\}$ satisfying

$$
d_{f}\left(\theta_{0}, \phi\right)=d\left(\theta_{0}\right) \sin 2\left(\phi-\theta_{0}\right), \quad n_{f}\left(\theta_{0}, \phi\right)=n\left(\theta_{0}\right) \sin 2\left(\phi-\theta_{0}\right) .
$$

Therefore for $\theta_{0} \in R_{f}^{G}$, there exists a number $\xi \in[0, \pi)$ satisfying (2.3) for any $\phi \in \boldsymbol{R}$.

Suppose now that there exists a number $\xi \in[0, \pi)$ satisfying (2.3) for any $\phi \in \boldsymbol{R}$, and $\operatorname{det}\left(\operatorname{Hess}_{f}\left(\theta_{0}\right)\right) \neq 0$. Then we obtain $\xi \neq \pi / 2$. Therefore it follows from Lemma 2.1, (2.2) and (2.4) that $(d \tilde{f} / d \theta)\left(\theta_{0}\right)=0$.

Hence we obtain Proposition 2.2.

The number $\xi \in[0, \pi)$ in Proposition 2.2 shall be denoted by $\xi\left(\theta_{0}\right)$.

For $\theta \in \boldsymbol{R}$, we set

$$
\mathrm{Umb}_{\theta}\left(\mathbf{G}_{f}\right):=\operatorname{Umb}\left(\mathbf{G}_{f}\right) \cap\{(\rho \cos \theta, \rho \sin \theta)\}_{\rho>0} .
$$

Then we obtain

COROllary 2.3. For $\theta_{0} \in \boldsymbol{R}$,

(1) $\operatorname{Hess}_{f}\left(\theta_{0}\right)$ is a scalar matrix if and only if $\theta_{0} \in R_{f}^{G}$ and $\xi\left(\theta_{0}\right)=0$ hold;

(2) $\operatorname{det}\left(\operatorname{Hess}_{f}\left(\theta_{0}\right)\right)=0$ holds if and only if $\theta_{0} \in R_{f}^{G}$ and $\xi\left(\theta_{0}\right)=\pi / 2$ hold;

(3) $\mathrm{Umb}_{\theta_{0}}\left(\mathrm{G}_{f}\right) \neq \varnothing$ holds if and only if $\theta_{0} \in R_{f}^{G}$ and $\xi\left(\theta_{0}\right) \in(0, \pi / 2)$ hold. If $\mathrm{Umb}_{\theta_{0}}\left(\mathrm{G}_{f}\right) \neq \emptyset$, then $\mathrm{Umb}_{\theta_{0}}\left(\mathrm{G}_{f}\right)=\left\{p\left(\theta_{0}\right)\right\}$ holds, where

$$
p\left(\theta_{0}\right):=\left(\rho\left(\theta_{0}\right) \cos \theta_{0}, \rho\left(\theta_{0}\right) \sin \theta_{0}\right), \quad \rho\left(\theta_{0}\right):=\left(\tan \xi\left(\theta_{0}\right)\right)^{1 /(2 k-2)} .
$$

COROLLARY 2.4. Let $\theta_{0}$ be an element of $\boldsymbol{R} \backslash R_{f}^{G}$ and $\phi_{0}$ a number satisfying (2.1) for some $\rho_{0}>0$. Then $d_{f}\left(\theta_{0}, \phi_{0}\right) n_{f}\left(\theta_{0}, \phi_{0}\right) \neq 0$ holds.

We shall prove

Proposition 2.5. $P_{F}^{k}=\left\{f \in P_{o}^{k} ; d \tilde{f} / d \theta \not \equiv 0\right\}$.

Proof. If $d \tilde{f} / d \theta \equiv 0$, then $k$ is even and $f$ is represented by $\left(x^{2}+y^{2}\right)^{k / 2}$ up to a constant ([1]). Then we obtain $d(\theta) n(\theta)<0$ for any $\theta \in \boldsymbol{R}$, where $d(\theta)$ and $n(\theta)$ are as in the proof of Proposition 2.2. Therefore we obtain $\xi(\theta) \in(0, \pi / 2)$ and $f \notin P_{F}^{k}$. If $d \tilde{f} / d \theta \not \equiv 0$, then the set $R_{f}^{G}$ is discrete. Corollary 2.3 implies that $\sharp \mathrm{Umb}_{\theta}\left(\mathrm{G}_{f}\right) \in\{0,1\}$. Therefore we obtain $f \in P_{F}^{k}$. Hence we have proved Proposition 2.5.

3. The behavior of the principal distributions on an open ray. From now on, we suppose $f \in P_{F}^{k}$. 
LEMMA 3.1. Let $\theta_{0}$ be an element of $\boldsymbol{R} \backslash R_{f}^{G}$ and $\xi$ an element of $[0, \pi / 2]$. Then there exists a number $\phi \in \boldsymbol{R}$ satisfying (2.3) and

$$
(\cos \xi) \frac{\partial d_{f}}{\partial \phi}\left(\theta_{0}, \phi\right)+(\sin \xi) \frac{\partial n_{f}}{\partial \phi}\left(\theta_{0}, \phi\right)=0
$$

if and only if $\left(\xi, \tilde{f}\left(\theta_{0}\right)\right)=(\pi / 2,0)$ holds.

Proof. There exists a matrix $Q_{f}\left(\xi, \theta_{0}\right)$ such that $\left\langle Q_{f}\left(\xi, \theta_{0}\right) u_{\phi}, u_{\phi}\right\rangle$ is equal to the left hand side of (2.3). Therefore there exists a number $\phi \in \boldsymbol{R}$ satisfying (2.3) and (3.1) if and only if $\operatorname{det}\left(Q_{f}\left(\xi, \theta_{0}\right)\right)=0$ holds.

Let $\eta_{\theta_{0}}$ be the number satisfying

$$
d_{f}\left(\theta_{0}, \eta_{\theta_{0}}\right)=0, \quad \eta_{\theta_{0}}<\theta_{0}<\eta_{\theta_{0}}+\pi / 2 .
$$

We set

$$
\lambda_{\theta_{0}}^{(1)}:=\left\langle\operatorname{Hess}_{f}\left(\theta_{0}\right) u_{\eta_{\theta_{0}}}, u_{\eta_{\theta_{0}}}\right\rangle, \quad \lambda_{\theta_{0}}^{(2)}:=\left\langle\operatorname{Hess}_{f}\left(\theta_{0}\right) u_{\eta_{\theta_{0}}+\pi / 2}, u_{\eta_{\theta_{0}}+\pi / 2}\right\rangle .
$$

Then for any $\phi \in \boldsymbol{R}$, the following holds:

$$
\begin{aligned}
n_{f}\left(\theta_{0}, \phi\right)=c_{k}\left(\theta_{0}\right) \sin \left(\phi-\theta_{0}\right)\{ & \lambda_{\theta_{0}}^{(1)} \cos \left(\phi-\eta_{\theta_{0}}\right) \cos \left(\theta_{0}-\eta_{\theta_{0}}\right) \\
& \left.+\lambda_{\theta_{0}}^{(2)} \sin \left(\phi-\eta_{\theta_{0}}\right) \sin \left(\theta_{0}-\eta_{\theta_{0}}\right)\right\} .
\end{aligned}
$$

Therefore we obtain

$$
n_{f}\left(\theta_{0}, \eta_{\theta_{0}}\right) \lambda_{\theta_{0}}^{(2)}<0, \quad n_{f}\left(\theta_{0}, \eta_{\theta_{0}}+\pi / 2\right) \lambda_{\theta_{0}}^{(1)}>0 .
$$

Similarly we obtain

$$
d_{f}\left(\theta_{0}, \theta_{0}\right)\left(\lambda_{\theta_{0}}^{(2)}-\lambda_{\theta_{0}}^{(1)}\right)>0 .
$$

It follows from (3.4) together with (3.5) that $\operatorname{det}\left(Q_{f}\left(\xi, \theta_{0}\right)\right)<0$ for any $\xi \in(0, \pi / 2)$.

We obtain $\operatorname{det}\left(Q_{f}\left(0, \theta_{0}\right)\right)<0$ and $\operatorname{det}\left(Q_{f}\left(\pi / 2, \theta_{0}\right)\right) \leqq 0$. For any $\theta \in \boldsymbol{R}$, the following holds:

$$
k(k-1) \tilde{f}(\theta)=\left\langle\operatorname{Hess}_{f}(\theta) u_{\theta}, u_{\theta}\right\rangle .
$$

Therefore we see that $\operatorname{det}\left(Q_{f}\left(\pi / 2, \theta_{0}\right)\right)=0$ is equivalent to $\theta_{0} \in Z_{f}^{\prime}$.

Hence we obtain Lemma 3.1.

Note that by Proposition 2.2 and Lemma 3.1, we obtain (1) of Proposition 1.1. Also, by (2.1) and Lemma 3.1, we obtain the following

LEMMA 3.2. For $\theta_{0} \notin R_{f}^{G}$ and for $\rho>0, \phi_{\omega, \theta_{0}}^{(i)}$ is smooth at $\rho$ and satisfies

$$
\frac{d \phi_{\omega, \theta_{0}}^{(i)}}{d \rho}(\rho)=\frac{2(k-1) d_{f}\left(\theta_{0}, \phi_{\omega, \theta_{0}}^{(i)}(\rho)\right)}{\left.\rho \frac{\partial}{\partial \phi}\left\{d_{f}+\rho^{2 k-2} n_{f}\right\}\right|_{\left(\theta_{0}, \phi_{\omega, \theta_{0}}^{(i)}(\rho)\right)}} .
$$


Proof of (2) of Proposition 1.1. For $\theta_{0} \in R_{f}^{G}$, (2) holds. If $\theta_{0} \notin R_{f}^{G}$, then from Corollary 2.4, we see that $\phi_{\omega, \theta_{0}}^{(i)}$ is bounded, and from Corollary 2.4 together with Lemma 3.2, we obtain $\left(d \phi_{\omega, \theta_{0}}^{(i)} / d \rho\right)(\rho) \neq 0$ for any $\rho>0$. Then (2) holds.

Let $\psi$ be a continuous function on $\boldsymbol{R}$ such that $\operatorname{grad}_{f}(\theta)$ is represented by $u_{\psi(\theta)}$ up to a constant for any $\theta \in \boldsymbol{R}$. Let $\Pi$ be the canonical projection from $\boldsymbol{R}$ onto $\boldsymbol{R} /\{n \pi ; n \in \boldsymbol{Z}\}$. Noticing (2.1), we obtain

LEMMA 3.3. The following hold:

(1) For any $\theta_{0} \in \boldsymbol{R}, u_{\phi_{\omega, o}^{(i)}\left(\theta_{0}\right)}$ is an eigenvector of $\operatorname{Hess}_{f}\left(\theta_{0}\right)$.

(2) For $\theta_{0} \notin R_{f}^{G},\left\{\Pi\left(\phi_{\omega, \infty}^{(i)}\left(\theta_{0}\right)\right)\right\}_{i=1}^{2}=\left\{\Pi\left(\theta_{0}\right), \Pi\left(\psi\left(\theta_{0}\right)+\pi / 2\right)\right\}$ holds.

By Lemma 3.1 and Lemma 3.3, we obtain (3) of Proposition 1.1.

LEMMA 3.4. For $\theta_{0} \notin R_{f}^{G}$, the following holds:

$$
\operatorname{det}\left(\operatorname{Hess}_{f}\left(\theta_{0}\right)\right) \frac{d \phi_{\omega, \theta_{0}}^{(1)}}{d \rho}(\rho) \frac{d \phi_{\omega, \theta_{0}}^{(2)}}{d \rho}(\rho)>0 .
$$

Proof. By Lemma 3.1, we obtain

$$
\left.\prod_{i=1}^{2} \frac{\partial}{\partial \phi}\left\{d_{f}+\rho^{2 k-2} n_{f}\right\}\right|_{\left(\theta_{0}, \phi_{\omega, \theta_{0}}^{(i)}(\rho)\right)}<0
$$

for any $\rho>0$. By (3.3), we obtain

$$
n_{f}\left(\theta_{0}, \phi_{\omega, o}^{(1)}\left(\theta_{0}\right)\right) n_{f}\left(\theta_{0}, \phi_{\omega, o}^{(2)}\left(\theta_{0}\right)\right) \operatorname{det}\left(\operatorname{Hess}_{f}\left(\theta_{0}\right)\right)<0 .
$$

Therefore by Corollary 2.4, we obtain

$$
n_{f}\left(\theta_{0}, \phi_{\omega, \theta_{0}}^{(1)}(\rho)\right) n_{f}\left(\theta_{0}, \phi_{\omega, \theta_{0}}^{(2)}(\rho)\right) \operatorname{det}\left(\operatorname{Hess}_{f}\left(\theta_{0}\right)\right)<0
$$

for any $\rho>0$. Hence Lemma 3.4 follows from (2.1), Lemma 3.2, (3.7) and (3.9).

4. An element of $\boldsymbol{Z}_{\boldsymbol{f}}$. From now on, for $\theta_{0} \in \boldsymbol{R}$ let $U_{\theta_{0}}$ be a neighborhood of $\theta_{0}$ in $\boldsymbol{R}$ satisfying

$$
U_{\theta_{0}} \backslash\left\{\theta_{0}\right\} \subset \boldsymbol{R} \backslash\left(R_{f}^{G} \cup Z_{f}\right) .
$$

LEMmA 4.1. It holds that $Z_{f}^{\prime}=Z_{f} \backslash R_{f}^{G}$ and $Z_{f}^{\prime \prime}=Z_{f} \cap R_{f}^{G}$. In addition,

(1) if $\theta_{1} \in Z_{f}^{\prime}$, then $\operatorname{det}\left(\operatorname{Hess}_{f}(\theta)\right)<0$ holds for any $\theta \in U_{\theta_{1}}$;

(2) if $\theta_{2} \in Z_{f}^{\prime \prime}$, then the following hold:

(a) $\operatorname{det}\left(\operatorname{Hess}_{f}\left(\theta_{2}\right)\right)=0$,

(b) $\operatorname{det}\left(\operatorname{Hess}_{f}(\theta)\right)<0$ for any $\theta \in U_{\theta_{2}} \backslash\left\{\theta_{2}\right\}$.

Proof. By (2.2), (2.4) and (3.6), we obtain $\operatorname{det}\left(\operatorname{Hess}_{f}\left(\theta_{1}\right)\right)<0$ for $\theta_{1} \in Z_{f}^{\prime}$. Then we obtain $Z_{f} \backslash R_{f}^{G}=Z_{f}^{\prime}$, and $Z_{f} \cap R_{f}^{G}=Z_{f}^{\prime \prime}$. If $\theta_{2} \in Z_{f}^{\prime \prime}$, then by (2.2), (2.4) and (3.6), we obtain $\operatorname{det}\left(\operatorname{Hess}_{f}\left(\theta_{2}\right)\right)=0$. We may represent $f$ as

$$
f(x, y)=\left\{-\left(\sin \theta_{2}\right) x+\left(\cos \theta_{2}\right) y\right\}^{2} g(x, y),
$$


where $g$ is a homogeneous polynomial satisfying $\theta_{2} \notin Z_{g}$. By a direct computation, we obtain $\operatorname{det}\left(\operatorname{Hess}_{f}(\theta)\right)<0$ for any $\theta \in U_{\theta_{2}} \backslash\left\{\theta_{2}\right\}$.

By (2.2), (2.4), (3.6) and (4.1), we obtain the following.

LeMma 4.2. For $\theta_{0} \in \boldsymbol{R}, \theta_{0} \in Z_{f}$ is equivalent to $\Pi\left(\theta_{0}\right)=\Pi\left(\psi\left(\theta_{0}\right)+\pi / 2\right)$.

LEMmA 4.3. Suppose $\theta_{0}=\psi\left(\theta_{0}\right)+\pi / 2$ for $\theta_{0} \in Z_{f}$. Then for any $\theta \in U_{\theta_{0}} \backslash\left\{\theta_{0}\right\}$, $(\theta-\psi(\theta)-\pi / 2)\left(\theta-\theta_{0}\right)>0$ holds.

We shall now prove

LEMMA 4.4. Let $\theta_{1}$ (resp. $\left.\theta_{2}\right)$ be an element of $Z_{f}^{\prime}\left(\right.$ resp. $\left.Z_{f}^{\prime \prime}\right)$. Then

(1) $\Gamma_{\omega_{1}, \omega_{2}}^{(i)}\left(\theta_{1}\right)=0$ holds;

(2) $\left\{\Gamma_{\omega, o}^{(i)}\left(\theta_{2}\right)\right\}_{i=1}^{2}=\{0\}$ and $\left\{\Gamma_{\omega, \infty}^{(i)}\left(\theta_{2}\right)\right\}_{i=1}^{2}=\{-\pi, 0\}$ hold.

In particular, $Z_{f} \cap S_{\omega}=\emptyset$ holds for $\omega=o, \infty, u$.

Proof. Suppose $\theta_{1} \in Z_{f}^{\prime}$. From Lemma 3.1, we obtain $\Gamma_{\omega, o}^{(i)}\left(\theta_{1}\right)=0$. Suppose $\phi_{\omega, o}^{(i)}\left(\theta_{1}\right)=\eta_{\theta_{1}}+(i-1) \pi / 2$, where $\eta_{\theta_{1}}$ is as in (3.2). Moreover, noticing Lemma 4.2, suppose $\theta_{1}=\psi\left(\theta_{1}\right)+\pi / 2$. Then by Corollary 2.4, Lemma 3.3, Lemma 3.4 and Lemma 4.1, we obtain

$$
\left\{\phi_{\omega, \infty}^{(1)}(\theta), \phi_{\omega, \infty}^{(2)}(\theta)\right\}=\{\theta, \psi(\theta)+\pi / 2\}
$$

for any $\theta \in U_{\theta_{1}}$. Therefore $\Gamma_{\omega, \infty}^{(i)}\left(\theta_{1}\right)=0$ holds. This proves (1).

Suppose $\theta_{2} \in Z_{f}^{\prime \prime}$. Then from Corollary 2.3 and Lemma 4.1, we obtain $\Gamma_{\omega, o}^{(i)}\left(\theta_{2}\right)=0$. Suppose $\theta_{2}=\psi\left(\theta_{2}\right)+\pi / 2$ and $\phi_{\omega, o}^{(1)}\left(\theta_{2}\right)=\psi\left(\theta_{2}\right)$. We then see that for any $\theta \in U_{\theta_{2}}, u_{\phi_{\omega, o}^{(1)}(\theta)}$ is an eigenvector of $\tilde{g}(\theta) \operatorname{Hess}_{f}(\theta)$ corresponding to the positive eigenvalue, where $g$ is as in (4.1). For $\theta_{0} \in \boldsymbol{R}$, we set

$$
U_{\theta_{0},+}:=U_{\theta_{0}} \cap\left\{\theta>\theta_{0}\right\}, \quad U_{\theta_{0},-}:=U_{\theta_{0}} \cap\left\{\theta<\theta_{0}\right\} .
$$

Then by Corollary 2.4, Lemma 3.4, Lemma 4.1 and Lemma 4.3, we obtain

$$
\phi_{\omega, \infty}^{(1)}(\theta)=\theta-\pi \quad(\text { resp. }=\theta)
$$

for any $\theta \in U_{\theta_{2},+}$ (resp. $\in U_{\theta_{2},-}$ ). Therefore $\Gamma_{\omega, \infty}^{(1)}\left(\theta_{2}\right)=-\pi$ holds. If $\phi_{\omega, o}^{(2)}\left(\theta_{2}\right)=\psi\left(\theta_{2}\right)+$ $\pi / 2$, then we obtain

$$
\phi_{\omega, \infty}^{(2)}(\theta)=\psi(\theta)+\pi / 2
$$

for any $\theta \in U_{\theta_{2}}$, and $\Gamma_{\omega, \infty}^{(2)}\left(\theta_{2}\right)=0$. This proves (2).

LEMMA 4.5. For $\omega \in\{o, \infty, u\}, S_{\omega} \subset R_{f}^{G}$ holds.

Proof. From Lemma 3.1, we obtain $S_{o} \cup S_{u} \subset R_{f}^{G}$. From Lemma 3.1 together with Lemma 4.4, we obtain $S_{\infty} \subset R_{f}^{G}$.

Noticing Corollary 2.3, Lemma 4.5 and that $\Pi\left(R_{f}^{G}\right)$ is a finite set, we obtain (1) of Proposition 1.2. 
Proposition 4.6. For $\theta_{0} \in \boldsymbol{R} \backslash Z_{f}^{\prime \prime}$ and for $\omega_{1}, \omega_{2} \in\{o, \infty\}$, there exists an integer $v_{\omega_{1}, \omega_{2}}\left(\theta_{0}\right)$ satisfying $\Gamma_{\omega_{1}, \omega_{2}}^{(i)}\left(\theta_{0}\right) \in I_{v_{\omega_{1}, \omega_{2}}\left(\theta_{0}\right)}$ for $i=1,2$.

Proof. By Lemma 3.3 and Lemma 4.2, we see that for $\omega_{1}, \omega_{2} \in\{o, \infty\}$, there exists an odd integer $n_{\omega_{1}, \omega_{2}}$ satisfying $\phi_{\omega_{1}, \omega_{2}}^{(2)}(\theta)-\phi_{\omega_{1}, \omega_{2}}^{(1)}(\theta) \in I_{n_{\omega_{1}, \omega_{2}}}$ for $\theta \in \boldsymbol{R} \backslash Z_{f}$. Noticing Lemma 4.4, we then obtain Proposition 4.6.

From Lemma 4.4 and Proposition 4.6, we obtain (2) of Proposition 1.2.

\section{The behavior of the principal distributions around $o$.}

LEMMA 5.1. Suppose that $\operatorname{Hess}_{f}\left(\theta_{0}\right)$ is a scalar matrix for $\theta_{0} \in \boldsymbol{R}$. Then $\eta\left(\theta_{0}\right) \notin$ $\left\{\theta_{0}+n \pi / 2\right\}_{n \in \boldsymbol{Z}}$ holds.

Proof. Suppose that $\operatorname{Hess}_{f}\left(\theta_{0}\right)$ is the unit matrix, and $\theta_{0}=0$. Then we may represent $f$ as $f(x, y):=\sum_{i=0}^{k} a_{i} x^{k-i} y^{i}$, where $a_{0}=1 / k(k-1), a_{1}=0$ and $a_{2}=1 / 2$. Therefore, $\operatorname{Hess}_{f}(\theta)=\sum_{i=0}^{k-2} M_{i} \cos ^{k-2-i} \theta \sin ^{i} \theta$ holds, where $M_{1}$ is not a diagonal matrix. Hence we obtain Lemma 5.1.

For $\theta_{0} \in R_{f}^{G}$, there exists a non-negative integer $m$ satisfying $\left(d^{m+1} \tilde{f} / d \theta^{m+1}\right)\left(\theta_{0}\right) \neq 0$. The minimum of such integers as $m$ is denoted by $\mu\left(\theta_{0}\right)$. Then $\theta_{0}$ is said to be related (resp. non-related) to the origin if $\mu\left(\theta_{0}\right)$ is odd (resp. even). If $\theta_{0}$ is related to the origin, then we say that the critical sign of $\theta_{0}$ is positive (resp. negative) if

$$
\tilde{f}\left(\theta_{0}\right) \frac{d^{\mu\left(\theta_{0}\right)+1} \tilde{f}}{d \theta^{\mu\left(\theta_{0}\right)+1}}\left(\theta_{0}\right) \leqq 0 \quad(\text { resp. }>0)
$$

holds, and denote the critical sign of $\theta_{0}$ by c-sign $\left(\theta_{0}\right)$. Suppose that $\theta_{0}$ is non-related to the origin. Then noticing (4.1), we obtain $\theta_{0} \notin Z_{f}$. The sign of a nonzero number $\tilde{f}\left(\theta_{0}\right)\left(d^{\mu\left(\theta_{0}\right)+1} \tilde{f} / d \theta^{\mu\left(\theta_{0}\right)+1}\right)\left(\theta_{0}\right)$ is denoted by $\operatorname{sign}\left(d_{\theta} \tilde{f}^{2}\left(\theta_{0}\right)\right)$. For $\theta_{0} \in R_{f}^{G}$, it occurs just one of the following cases:

$$
\begin{array}{ll}
\mathrm{c}-\operatorname{sign}\left(\theta_{0}\right)=+, & \operatorname{sign}\left(d_{\theta} \tilde{f}^{2}\left(\theta_{0}\right)\right)=+, \\
\mathrm{c}-\operatorname{sign}\left(\theta_{0}\right)=-, & \operatorname{sign}\left(d_{\theta} \tilde{f}^{2}\left(\theta_{0}\right)\right)=-.
\end{array}
$$

Lemma 5.2. Suppose that $\operatorname{Hess}_{f}\left(\theta_{0}\right)$ is a scalar matrix for $\theta_{0} \in \boldsymbol{R}$. Then $\theta_{0} \in R_{f}^{G}$ and c-sign $\left(\theta_{0}\right)=-$ hold.

Proof. By (2.2), (2.4) and (3.6), we obtain Lemma 5.2.

Lemma 5.3. Let $\theta_{0}$ be an element of $\boldsymbol{R} \backslash\left(R_{f}^{G} \cup Z_{f}\right)$. Suppose that $\Pi\left(\theta_{0}\right)=$ $\Pi\left(\phi_{\omega, \infty}^{\left(i_{0}\right)}\left(\theta_{0}\right)\right)$ holds for $i_{0} \in\{1,2\}$. Then the following holds:

$$
\frac{d \phi_{\omega, \theta_{0}}^{\left(i_{0}\right)}}{d \rho}(\rho) \tilde{f}\left(\theta_{0}\right) \frac{d \tilde{f}}{d \theta}\left(\theta_{0}\right) \operatorname{det}\left(\operatorname{Hess}_{f}\left(\theta_{0}\right)\right)>0 .
$$

PROOF. By Corollary 2.4, Lemma 3.1 and Lemma 3.2, we obtain

$$
\frac{d \phi_{\omega, \theta_{0}}^{\left(i_{0}\right)}}{d \rho}(\rho) d_{f}\left(\theta_{0}, \phi_{\omega, \infty}^{\left(i_{0}\right)}\left(\theta_{0}\right)\right) \frac{\partial n_{f}}{\partial \phi}\left(\theta_{0}, \phi_{\omega, \infty}^{\left(i_{0}\right)}\left(\theta_{0}\right)\right) \geqq 0 .
$$


On the other hand, the following holds:

$$
d_{f}\left(\theta_{0}, \phi_{\omega, \infty}^{\left(i_{0}\right)}\left(\theta_{0}\right)\right) \frac{\partial n_{f}}{\partial \phi}\left(\theta_{0}, \phi_{\omega, \infty}^{\left(i_{0}\right)}\left(\theta_{0}\right)\right)=k \tilde{f}\left(\theta_{0}\right) \frac{d \tilde{f}}{d \theta}\left(\theta_{0}\right) \operatorname{det}\left(\operatorname{Hess}_{f}\left(\theta_{0}\right)\right) .
$$

Hence we obtain Lemma 5.3.

LEMMA 5.4. Suppose that $\operatorname{Hess}_{f}\left(\theta_{0}\right)$ is a scalar matrix for $\theta_{0} \in \boldsymbol{R}$. Then for any $\theta \in U_{\theta_{0}} \backslash\left\{\theta_{0}\right\},\left(d \phi_{\omega, \theta}^{(i)} / d \rho\right)(\rho)\left(\theta-\theta_{0}\right)>0$ holds.

Proof. Noticing Corollary 2.3, we suppose $\phi_{\omega, \infty}^{(1)}(\theta)=\theta$ for any $\theta \in U_{\theta_{0}}$. Then by Lemma 5.2 and Lemma 5.3, we obtain $\left(d \phi_{\omega, \theta}^{(1)} / d \rho\right)(\rho)\left(\theta-\theta_{0}\right)>0$ for any $\theta \in U_{\theta_{0}} \backslash\left\{\theta_{0}\right\}$. By Lemma 3.4, we also obtain $\left(d \phi_{\omega, \theta}^{(2)} / d \rho\right)(\rho)\left(\theta-\theta_{0}\right)>0$. Hence we have Lemma 5.4.

Proof of (1) of Theorem 1.3. If $\operatorname{Hess}_{f}\left(\theta_{0}\right)$ is a scalar matrix for $\theta_{0} \in \boldsymbol{R}$, then by Lemma 5.1 and Lemma 5.4, we obtain $\Gamma_{o, o}^{(i)}\left(\theta_{0}\right)=-\pi / 2, \theta_{0} \in S_{o}$ and $v_{o, o}\left(\theta_{0}\right)=-1$. It follows from Corollary 2.3 together with Lemma 4.5 that $\operatorname{Hess}_{f}\left(\theta_{0}\right)$ is a scalar matrix for $\theta_{0} \in S_{o}$. This proves (a) and (b).

Note that for any $\theta \in \boldsymbol{R}$, the following holds:

$$
\operatorname{ind}_{o}\left(\tilde{\mathbf{G}}_{f}\right)=\frac{\phi_{r_{o}}^{(i)}(\theta+2 \pi)-\phi_{r_{o}}^{(i)}(\theta)}{2 \pi} .
$$

By Lemma 3.1 and Lemma 3.3, we obtain

$$
\phi_{r_{o}}^{(i)}(\theta+2 \pi)-\phi_{r_{o}}^{(i)}(\theta)=\eta(\theta+2 \pi)-\eta(\theta)+\sum_{\theta_{0} \in S_{o} \cap[\theta, \theta+2 \pi)} \Gamma_{o, o}^{(i)}\left(\theta_{0}\right) .
$$

By $\Gamma_{o, o}^{(i)}\left(\theta_{0}\right)=-\pi / 2$ for $\theta_{0} \in S_{o},(5.1)$ and (5.2), we then obtain (c).

REMARK. Let $R\left(\operatorname{Hess}_{f}\right)$ be the set of the numbers such that each $\theta_{0} \in R\left(\operatorname{Hess}_{f}\right)$ satisfies $\eta\left(\theta_{0}\right) \in\left\{\theta_{0}+n \pi / 2\right\}_{n \in Z}$. By (2.2) and Lemma 5.1, we obtain $R\left(\operatorname{Hess}_{f}\right) \subset R_{f}^{G} \backslash S_{o}$. We say that the sign of $\theta_{0} \in R\left(\right.$ Hess $\left._{f}\right)$ is positive (resp. negative) if there exists a neighborhood $U_{\theta_{0}}$ of $\theta_{0}$ in $\boldsymbol{R}$ satisfying

$$
\left\{\theta-\eta(\theta)-\left(\theta_{0}-\eta\left(\theta_{0}\right)\right)\right\}\left(\theta-\theta_{0}\right)>0 \quad(\text { resp. }<0)
$$

for any $\theta \in U_{\theta_{0}} \backslash\left\{\theta_{0}\right\}$. When this is the case, the sign is denoted by $\operatorname{sign}\left(\theta_{0}\right)$. For $\sigma \in\{+,-\}$, if we set

$$
n_{\sigma}:=\sharp \Pi\left(\left\{\theta_{0} \in R\left(\operatorname{Hess}_{f}\right) ; \operatorname{sign}\left(\theta_{0}\right)=\sigma\right\}\right),
$$

then we obtain

$$
\frac{\eta(\theta+2 \pi)-\eta(\theta)}{2 \pi}=1-\frac{n_{+}-n_{-}}{2} .
$$

For $\theta_{0} \in R\left(\operatorname{Hess}_{f}\right),\left(\partial d_{f} / \partial \phi\right)\left(\theta_{0}, \eta\left(\theta_{0}\right)\right) \neq 0$ holds. Therefore, by the implicit function theorem, we see that $\eta$ is infinitely differentiable at $\theta_{0}$. By

$$
d_{f}(\theta, \theta)=(k-1) \frac{d \tilde{f}}{d \theta}(\theta),
$$


we obtain

$$
\frac{d^{m}(\theta-\eta)}{d \theta^{m}}\left(\theta_{0}\right)=(k-1) \frac{d^{m+1} \tilde{f}}{d \theta^{m+1}}\left(\theta_{0}\right) / \frac{\partial d_{f}}{\partial \phi}\left(\theta_{0}, \theta_{0}\right)
$$

for $m=1, \ldots, \mu\left(\theta_{0}\right)$. Therefore we see that for $\theta_{0} \in R\left(\operatorname{Hess}_{f}\right)$, the sign of $\theta_{0}$ is positive or negative if and only if $\theta_{0}$ is related to the origin. Also, if $\theta_{0}$ is related to the origin, then $\operatorname{sign}\left(\theta_{0}\right)$ is given by the sign of the nonzero number

$$
\delta\left(\theta_{0}\right):=\frac{d^{\mu\left(\theta_{0}\right)+1} \tilde{f}}{d \theta^{\mu\left(\theta_{0}\right)+1}}\left(\theta_{0}\right) \frac{\partial d_{f}}{\partial \phi}\left(\theta_{0}, \theta_{0}\right) .
$$

This number has been studied in [2]. For $\theta_{0} \in R\left(\operatorname{Hess}_{f}\right)$ related to the origin, $\mathrm{c}-\operatorname{sign}\left(\theta_{0}\right)=$ + implies $\delta\left(\theta_{0}\right)>0$; if $\mathrm{c}-\operatorname{sign}\left(\theta_{0}\right)=-$, then $\delta\left(\theta_{0}\right)>0$ (resp. $<0$ ) is equivalent to $\mathrm{Umb}_{\theta_{0}}\left(\mathrm{G}_{f}\right)=\emptyset($ resp. $\neq \varnothing)$.

6. The behavior of the principal distributions around $\infty$. An element $\theta_{0} \in R_{f}^{G}$ is said to be related (resp. non-related) to the curvature if there exists a nonzero number $c\left(\theta_{0}\right)$ satisfying $c\left(\theta_{0}\right) \operatorname{det}\left(\operatorname{Hess}_{f}(\theta)\right)\left(\theta-\theta_{0}\right)^{m}>0$ for any $\theta \in U_{\theta_{0}} \backslash\left\{\theta_{0}\right\}$ and for $m=1$ (resp. $=0$ ). If $\theta_{0}$ is related (resp. non-related) to the curvature, then the sign of $c\left(\theta_{0}\right)$ is denoted by k-sign $\left(\theta_{0}\right)\left(\right.$ resp. $\left.\operatorname{sign}\left[\tilde{K}_{f}\left(\theta_{0}\right)\right]\right)$. For $\theta_{0} \in R_{f}^{G}$, it occurs just one of the following cases:

$$
\begin{array}{ll}
\mathrm{k}-\operatorname{sign}\left(\theta_{0}\right)=+, & \operatorname{sign}\left[\tilde{K}_{f}\left(\theta_{0}\right)\right]=+, \\
\mathrm{k}-\operatorname{sign}\left(\theta_{0}\right)=-, & \operatorname{sign}\left[\tilde{K}_{f}\left(\theta_{0}\right)\right]=-.
\end{array}
$$

Let denote the law of composition of the set $\{+,-\}$ of symbols,+- satisfying $+\cdot+=$ $-\cdot-=+$ and $+\cdot-=-\cdot+=-$.

PROPOSITION 6.1. Let $\theta_{0}$ be a number satisfying $\operatorname{det}\left(\operatorname{Hess}_{f}\left(\theta_{0}\right)\right)=0$.

(1) If $\theta_{0}$ is related to the origin and satisfies $\theta_{0} \notin Z_{f}$, then the following holds:

$$
v_{\infty, \infty}\left(\theta_{0}\right)= \begin{cases}0 & \text { if } \mathrm{c}-\operatorname{sign}\left(\theta_{0}\right) \cdot \operatorname{sign}\left[\tilde{K}_{f}\left(\theta_{0}\right)\right]=-, \\ -1 & \text { if } \theta_{0} \text { is related to the curvature }, \\ -2 & \text { if } \mathrm{c}-\operatorname{sign}\left(\theta_{0}\right) \cdot \operatorname{sign}\left[\tilde{K}_{f}\left(\theta_{0}\right)\right]=+.\end{cases}
$$

(2) If $\theta_{0}$ is non-related to the origin, then the following holds:

$$
v_{\infty, \infty}\left(\theta_{0}\right)= \begin{cases}1 & \text { if } \operatorname{sign}\left(d_{\theta} \tilde{f}^{2}\left(\theta_{0}\right)\right) \cdot \mathrm{k}-\operatorname{sign}\left(\theta_{0}\right)=+, \\ 0 & \text { if } \theta_{0} \text { is non-related to the curvature, } \\ -1 & \text { if } \operatorname{sign}\left(d_{\theta} \tilde{f}^{2}\left(\theta_{0}\right)\right) \cdot \mathrm{k}-\operatorname{sign}\left(\theta_{0}\right)=-.\end{cases}
$$

Proof. Suppose $(d \tilde{f} / d \theta)\left(\theta_{0}\right)=0$ and $\theta_{0} \notin Z_{f}$. Then we obtain $\tilde{f}\left(\theta_{0}\right)\left(d^{2} \tilde{f} / d \theta^{2}\right)\left(\theta_{0}\right)$ $<0$. Therefore by Lemma 5.3, we see that if $i_{0} \in\{1,2\}$ and $\theta \in U_{\theta_{0}} \backslash\left\{\theta_{0}\right\}$ satisfy $\Pi\left(\phi_{\infty, \infty}^{\left(i_{0}\right)}(\theta)\right)=\Pi(\theta)$, then the following holds:

$$
\frac{d \phi_{\infty, \theta}^{\left(i_{0}\right)}}{d \rho}(\rho) \operatorname{det}\left(\operatorname{Hess}_{f}(\theta)\right)\left(\theta-\theta_{0}\right)<0 .
$$


Suppose that $\theta_{0}=\psi\left(\theta_{0}\right)=\phi_{\infty, o}^{(1)}\left(\theta_{0}\right)$ and $\phi_{\infty, o}^{(2)}\left(\theta_{0}\right)=\theta_{0}+\pi / 2$. Then by (3.8), we obtain

$$
\operatorname{det}\left(\operatorname{Hess}_{f}(\theta)\right) \sin \left(\theta-\phi_{\infty, o}^{(1)}(\theta)\right) \sin \left(\phi_{\infty, o}^{(1)}(\theta)-\psi(\theta)\right)<0
$$

for any $\theta \in U_{\theta_{0}} \backslash\left\{\theta_{0}\right\}$. Noticing that $u_{\theta_{0}}$ is an eigenvector of $\operatorname{Hess}_{f}\left(\theta_{0}\right)$ corresponding to the nonzero eigenvalue, we see the following:

(1) If $\theta \in U_{\theta_{0},+}$ satisfies $\operatorname{det}\left(\operatorname{Hess}_{f}(\theta)\right)>0$ (resp. $<0$ ), then the following holds:

$$
\left.\phi_{\infty, o}^{(1)}(\theta)<\psi(\theta)<\theta \quad \text { (resp. } \psi(\theta)<\phi_{\infty, o}^{(1)}(\theta)<\theta\right) .
$$

(2) If $\theta \in U_{\theta_{0},-}$ satisfies $\operatorname{det}\left(\operatorname{Hess}_{f}(\theta)\right)>0$ (resp. $<0$ ), then the following holds:

$$
\theta<\psi(\theta)<\phi_{\infty, o}^{(1)}(\theta) \quad\left(\text { resp. } \theta<\phi_{\infty, o}^{(1)}(\theta)<\psi(\theta)\right) .
$$

Then by (6.1), we obtain (1) of Proposition 6.1.

Suppose $(d \tilde{f} / d \theta)\left(\theta_{0}\right) \neq 0$. Then for any $\theta \in U_{\theta_{0}}$, the following holds:

$$
\Pi(\theta) \notin\left\{\Pi(\psi(\theta)), \Pi(\psi(\theta)+\pi / 2), \Pi\left(\phi_{\infty, o}^{(1)}(\theta)\right), \Pi\left(\phi_{\infty, o}^{(2)}(\theta)\right)\right\} .
$$

Suppose that $\theta_{0}$ is related to the curvature. Then by Lemma 5.3, we see that if $i_{0} \in\{1,2\}$ and $\theta \in U_{\theta_{0}} \backslash\left\{\theta_{0}\right\}$ satisfy $\Pi\left(\phi_{\infty, \infty}^{\left(i_{0}\right)}(\theta)\right)=\Pi(\theta)$, then the sign of $\left(d \phi_{\infty, \theta}^{\left(i_{0}\right)} / d \rho\right)(\rho)\left(\theta-\theta_{0}\right)$ is given by $\operatorname{sign}\left(d_{\theta} \tilde{f}^{2}\left(\theta_{0}\right)\right) \cdot \mathrm{k}$-sign $\left(\theta_{0}\right)$. Therefore noticing (6.2), we see that if $\Pi\left(\phi_{\infty, \infty}^{(1)}\left(\theta_{-}\right)\right)=$ $\Pi\left(\theta_{-}\right)$holds for $\theta_{-} \in U_{\theta_{0},-}$, then $\Pi\left(\phi_{\infty, \infty}^{(1)}\left(\theta_{+}\right)\right)=\Pi\left(\psi\left(\theta_{+}\right)+\pi / 2\right)$ holds for $\theta_{+} \in U_{\theta_{0},+}$. Hence we obtain

$$
v_{\infty, \infty}\left(\theta_{0}\right)= \begin{cases}1 & \text { if } \operatorname{sign}\left(d_{\theta} \tilde{f}^{2}\left(\theta_{0}\right)\right) \cdot \mathrm{k}-\operatorname{sign}\left(\theta_{0}\right)=+, \\ -1 & \text { if } \operatorname{sign}\left(d_{\theta} \tilde{f}^{2}\left(\theta_{0}\right)\right) \cdot \mathrm{k}-\operatorname{sign}\left(\theta_{0}\right)=-.\end{cases}
$$

If $\theta_{0}$ is non-related to the curvature, then we obtain $\Gamma_{\infty, \infty}^{(i)}\left(\theta_{0}\right)=0$. Consequently, we obtain (2) of Proposition 6.1.

Next, we shall prove

LEMMA 6.2. For $\theta_{0} \in \boldsymbol{R}, i_{0}=1$ or 2 satisfies the condition

$$
\text { (*) } \begin{cases}\Pi\left(\phi_{\infty, \infty}^{\left(i_{0}\right)}(\theta)\right)=\Pi(\theta) & \text { for any } \theta \in U_{\theta_{0},-}, \\ \Pi\left(\phi_{\infty, \infty}^{\left(i_{0}\right)}(\theta)\right)=\Pi(\psi(\theta)+\pi / 2) & \text { for any } \theta \in U_{\theta_{0},+}\end{cases}
$$

if and only if one of the following holds:

(1) A number $\theta_{0}$ is an element of $Z_{f}^{\prime}$.

(2) A number $\theta_{0}$ is an element of $S_{\infty}$ such that $v_{\infty, \infty}\left(\theta_{0}\right)$ is odd.

Proof. By Lemma 4.3 together with (4.2), we see that $i_{0}=1$ or 2 satisfies condition (*) for $\theta_{0} \in Z_{f}^{\prime}$. From (4.3), we also see that $i_{0} \in\{1,2\}$ does not satisfy (*) for $\theta_{0} \in Z_{f}^{\prime \prime}$. By Lemma 4.2, we then see that $i_{0}=1$ or 2 satisfies $(*)$ for $\theta_{0} \notin Z_{f}$ if and only if $\theta_{0}$ is an element of $S_{\infty}$ such that $v_{\infty, \infty}$ is odd. Hence we have Lemma 6.2.

LEMMA 6.3. Let $\tilde{\theta}_{1}, \tilde{\theta}_{2}$ be numbers satisfying
(1) $\tilde{\theta}_{i} \notin R_{f}^{G}$ for $i=1,2$,
(2) $\tilde{\theta}_{1}<\tilde{\theta}_{2}$
(3) $\operatorname{det}\left(\operatorname{Hess}_{f}\left(\tilde{\theta}_{i}\right)\right)<0 \quad$ for $i=1,2$,
(4) $\left(\tilde{\theta}_{1}, \tilde{\theta}_{2}\right) \cap Z_{f}^{\prime}=\emptyset$. 
Then the following holds:

$$
\begin{aligned}
\left\{\phi_{\infty, \infty}^{(i)}\right. & \left.\left(\tilde{\theta}_{2}\right)-\phi_{\infty, \infty}^{(i)}\left(\tilde{\theta}_{1}\right)\right\}_{i=1}^{2} \\
& =\left\{\tilde{\theta}_{2}-\tilde{\theta}_{1}-\zeta_{f}^{\prime \prime}\left(\tilde{\theta}_{1}, \tilde{\theta}_{2}\right) \pi+\frac{\pi}{2} v_{\infty}\left(\tilde{\theta}_{1}, \tilde{\theta}_{2}\right), \psi\left(\tilde{\theta}_{2}\right)-\psi\left(\tilde{\theta}_{1}\right)+\frac{\pi}{2} v_{\infty}\left(\tilde{\theta}_{1}, \tilde{\theta}_{2}\right)\right\}
\end{aligned}
$$

where

$$
\zeta_{f}^{\prime \prime}\left(\tilde{\theta}_{1}, \tilde{\theta}_{2}\right):=\sharp\left[Z_{f}^{\prime \prime} \cap\left(\tilde{\theta}_{1}, \tilde{\theta}_{2}\right)\right], \quad v_{\infty}\left(\tilde{\theta}_{1}, \tilde{\theta}_{2}\right):=\sum_{\theta_{0} \in S_{\infty} \cap\left(\tilde{\theta}_{1}, \tilde{\theta}_{2}\right)} v_{\infty, \infty}\left(\theta_{0}\right)
$$

Proof. From Corollary 2.3 and Lemma 4.5, we obtain $\operatorname{det}\left(\operatorname{Hess}_{f}\left(\theta_{0}\right)\right)=0$ for $\theta_{0} \in$ $S_{\infty}$. Therefore by Lemma 4.1, (4.3), (4.4), Proposition 6.1 and Lemma 6.2, we obtain Lemma 6.3 .

Proof of (2) of Theorem 1.3. We know $\operatorname{det}\left(\operatorname{Hess}_{f}\left(\theta_{0}\right)\right)=0$ for $\theta_{0} \in S_{\infty}$. From Lemma 4.4 and Proposition 6.1, we obtain $v_{\infty, \infty}\left(\theta_{0}\right) \in\{1,-1,-2\}$ for $\theta_{0} \in S_{\infty}$. Hence we obtain (a).

For any $\theta \in \boldsymbol{R}$, the following holds:

$$
\operatorname{ind}_{\infty}\left(\tilde{\mathbf{G}}_{f}\right)=2-\frac{\phi_{r_{\infty}}^{(i)}(\theta+2 \pi)-\phi_{r_{\infty}}^{(i)}(\theta)}{2 \pi}
$$

Suppose $Z_{f}^{\prime}=\emptyset$. Then by Lemma 6.3, we obtain

$$
\begin{aligned}
\phi_{r_{\infty}}^{(i)}\left(\tilde{\theta}_{0}+2 \pi\right)-\phi_{r_{\infty}}^{(i)}\left(\tilde{\theta}_{0}\right) \\
\quad=\frac{1}{2}\left\{2 \pi-\zeta_{f}^{\prime \prime}\left(\tilde{\theta}_{0}, \tilde{\theta}_{0}+2 \pi\right) \pi+\psi\left(\tilde{\theta}_{0}+2 \pi\right)-\psi\left(\tilde{\theta}_{0}\right)+\pi v_{\infty}\left(\tilde{\theta}_{0}, \tilde{\theta}_{0}+2 \pi\right)\right\}
\end{aligned}
$$

for any $\tilde{\theta}_{0} \in \boldsymbol{R} \backslash R_{f}^{G}$. Noticing $(6.3),(6.4), \zeta_{f}^{\prime \prime}\left(\tilde{\theta}_{0}, \tilde{\theta}_{0}+2 \pi\right)=2 \sharp \Pi\left(Z_{f}^{\prime \prime}\right)$ and

$$
\frac{\psi\left(\tilde{\theta}_{0}+2 \pi\right)-\psi\left(\tilde{\theta}_{0}\right)}{2 \pi}=1-\sharp \Pi\left(Z_{f}\right),
$$

we obtain (b).

Suppose $Z_{f}^{\prime} \neq \emptyset$. Let $\left\{\tilde{\theta}_{i}\right\}_{i=0}^{2 \sharp \Pi\left(Z_{f}^{\prime}\right)}$ be a subset of $Z_{f}^{\prime}$ satisfying

$$
\tilde{\theta}_{0}<\tilde{\theta}_{1}<\cdots<\tilde{\theta}_{2 \sharp \Pi\left(Z_{f}^{\prime}\right)-1}<\tilde{\theta}_{2 \sharp \Pi\left(Z_{f}^{\prime}\right)}=\tilde{\theta}_{0}+2 \pi .
$$


Then by Lemma 4.1, Lemma 6.2 and Lemma 6.3, we see that

$$
\begin{aligned}
&\left\{\phi_{\infty}^{(i)}\left(\tilde{\theta}_{0}+2 \pi\right)-\phi_{\infty}^{(i)}\left(\tilde{\theta}_{0}\right)\right\}_{i=1}^{2} \\
&=\left\{\sum_{i=1}^{\sharp \Pi\left(Z_{f}^{\prime}\right)}\left(\tilde{\theta}_{2 i}-\tilde{\theta}_{2 i-1}-\zeta_{f}^{\prime \prime}\left(\tilde{\theta}_{2 i-1}, \tilde{\theta}_{2 i}\right) \pi\right)\right. \\
&+\sum_{i=1}^{\sharp \Pi\left(Z_{f}^{\prime}\right)}\left(\psi\left(\tilde{\theta}_{2 i-1}\right)-\psi\left(\tilde{\theta}_{2 i-2}\right)\right)+\frac{\pi}{2} v_{\infty}\left(\tilde{\theta}_{0}, \tilde{\theta}_{0}+2 \pi\right), \\
& \quad \sum_{i=1} \sum_{\left.Z_{f}^{\prime}\right)}\left(\tilde{\theta}_{2 i-1}-\tilde{\theta}_{2 i-2}-\zeta_{f}^{\prime \prime}\left(\tilde{\theta}_{2 i-2}, \tilde{\theta}_{2 i-1}\right) \pi\right) \\
&\left.+\sum_{i=1}^{\sharp \Pi\left(Z_{f}^{\prime}\right)}\left(\psi\left(\tilde{\theta}_{2 i}\right)-\psi\left(\tilde{\theta}_{2 i-1}\right)\right)+\frac{\pi}{2} v_{\infty}\left(\tilde{\theta}_{0}, \tilde{\theta}_{0}+2 \pi\right)\right\} .
\end{aligned}
$$

Therefore we obtain (6.4), and (b).

7. The behavior of the principal distributions around an umbilical point on an open ray. If $\theta_{0} \in S_{u}$, then from Corollary 2.3 together with Lemma 4.5, we obtain $\mathrm{Umb}_{\theta_{0}}\left(\mathrm{G}_{f}\right) \neq \emptyset$. Generally, if $\mathrm{Umb}_{\theta_{0}}\left(\mathrm{G}_{f}\right) \neq \emptyset$, then the following hold:

$$
\operatorname{ind}_{p\left(\theta_{0}\right)}\left(\tilde{\mathrm{G}}_{f}\right)=\frac{\Gamma_{\infty, o}^{(i)}\left(\theta_{0}\right)}{2 \pi}=-\frac{\Gamma_{o, \infty}^{(i)}\left(\theta_{0}\right)}{2 \pi} .
$$

Hence we obtain (a) of (3) of Theorem 1.3. To prove (b), we have only to see

PROPOSITION 7.1. If $\mathrm{Umb}_{\theta_{0}}\left(\mathrm{G}_{f}\right) \neq \emptyset$, then the following holds:

$$
\Gamma_{\infty, o}^{(i)}\left(\theta_{0}\right)= \begin{cases}\pi & \text { if } \mathrm{c}-\operatorname{sign}\left(\theta_{0}\right)=+ \\ 0 & \text { if } \theta_{0} \text { is non-related to the origin }, \\ -\pi & \text { if } \mathrm{c}-\operatorname{sign}\left(\theta_{0}\right)=-.\end{cases}
$$

To prove Proposition 7.1, we need the following lemmas.

LEMMA 7.2. For $\theta_{0} \in \boldsymbol{R}, \mathrm{Umb}_{\theta_{0}}\left(\mathrm{G}_{f}\right) \neq \varnothing$ is equivalent to $\Pi\left(\phi_{o, \theta_{0}}^{(i)}(\rho)\right) \neq$ $\Pi\left(\phi_{\infty, \theta_{0}}^{(i)}(\rho)\right)$ for some $\rho>0$.

Proof. We obtain $\operatorname{Umb}_{\theta_{0}}\left(\mathrm{G}_{f}\right) \neq \emptyset$ from $\Pi\left(\phi_{o, \theta_{0}}^{(i)}(\rho)\right) \neq \Pi\left(\phi_{\infty, \theta_{0}}^{(i)}(\rho)\right)$.

If $\mathrm{Umb}_{\theta_{0}}\left(\mathrm{G}_{f}\right) \neq \emptyset$, then by Corollary 2.3, we see that there exists a nonzero number $c_{\omega}^{(i)}\left(\theta_{0}\right) \neq 0$ satisfying

$$
\left.c_{\omega}^{(i)}\left(\theta_{0}\right)\left(\rho-\rho\left(\theta_{0}\right)\right) \frac{\partial}{\partial \phi}\left\{d_{f}+\rho^{2 k-2} n_{f}\right\}\right|_{\left(\theta_{0}, \phi_{\omega, \theta_{0}}^{(i)}(\rho)\right)}>0
$$


for $\rho \neq \rho\left(\theta_{0}\right)$. On the other hand, we obtain $r_{o}<\rho\left(\theta_{0}\right)<r_{\infty}$ and

$$
\left.\prod_{\omega \in\{0, \infty\}} \frac{\partial}{\partial \phi}\left\{d_{f}+r_{\omega}^{2 k-2} n_{f}\right\}\right|_{\left(\theta_{0}, \phi_{\omega, \theta_{0}}^{(i)}\left(r_{\omega}\right)\right)}>0 .
$$

Therefore $\Pi\left(\phi_{o, \theta_{0}}^{(i)}\left(r_{\omega}\right)\right) \neq \Pi\left(\phi_{\infty, \theta_{0}}^{(i)}\left(r_{\omega}\right)\right)$ holds.

LEMMA 7.3. If $\operatorname{Umb}_{\theta_{0}}\left(\mathrm{G}_{f}\right) \neq \emptyset$, then $\operatorname{det}\left(\operatorname{Hess}_{f}\left(\theta_{0}\right)\right)>0$ holds.

Proof. Corollary 2.3 implies that $\operatorname{det}\left(\operatorname{Hess}_{f}\left(\theta_{0}\right)\right) \neq 0$. If $\operatorname{det}\left(\operatorname{Hess}_{f}\left(\theta_{0}\right)\right)<0$, then we obtain $d\left(\theta_{0}\right) n\left(\theta_{0}\right)>0$, where $d\left(\theta_{0}\right)$ and $n\left(\theta_{0}\right)$ are as in the proof of Proposition 2.2. This contradicts $\xi\left(\theta_{0}\right) \in(0, \pi / 2)$.

Proof of Proposition 7.1. By Corollary 2.3, we see that $i_{0}=1$ or 2 satisfies $\Pi\left(\phi_{\infty, \infty}^{\left(i_{0}\right)}(\theta)\right)=\Pi(\theta)$ for any $\theta \in U_{\theta_{0}}$. By Lemma 5.3 and Lemma 7.3, we see that if c-sign $\left(\theta_{0}\right)=+($ resp. $=-)$, then $\left(d \phi_{\infty, \theta}^{\left(i_{0}\right)} / d \rho\right)(\rho)\left(\theta_{0}-\theta\right)>0$ (resp. $\left.<0\right)$ holds for any $\theta \in U_{\theta_{0}} \backslash\left\{\theta_{0}\right\}$, and that if $\theta_{0}$ is non-related to the origin, then there exists a nonzero number $\hat{c}\left(\theta_{0}\right)$ satisfying $\hat{c}\left(\theta_{0}\right)\left(d \phi_{\infty, \theta}^{\left(i_{0}\right)} / d \rho\right)(\rho)>0$ for any $\theta \in U_{\theta_{0}} \backslash\left\{\theta_{0}\right\}$. Then, noticing Lemma 7.2 and

$$
\Pi\left(\phi_{\infty, o}^{\left(i_{0}\right)}\left(\theta_{0}+0\right)\right)=\Pi\left(\phi_{\infty, o}^{\left(i_{0}\right)}\left(\theta_{0}-0\right)\right)=\Pi\left(\phi_{o, o}^{\left(i_{0}\right)}\left(\theta_{0}\right)\right),
$$

we obtain Proposition 7.1.

\section{REFERENCES}

[1] N. ANDo, An isolated umbilical point of the graph of a homogeneous polynomial, Geom. Dedicata 82 (2000), $115-137$.

[2 ] N. ANDO, The behavior of the principal distributions around an isolated umbilical point, J. Math. Soc. Japan 53 (2001), 237-260.

[ 3 ] C. Gutierrez and F. SAnChez-Bringas, Planer vector field versions of Carathéodory's and Loewner's conjectures, Publ. Mat. 41 (1997), 169-179.

[ 4 ] P. Hartman And A. Wintner, Umbilical points and W-surfaces, Amer. J. Math. 76 (1954), 502-508.

[ 5 ] H. Hopf, Lectures on differential geometry in the large, Lecture Notes in Math. vol. 1000, Springer-Verlag, Berlin-New York, 1989.

[ 6 ] T. KLOTZ, On Bol's proof of Carathéodory's conjecture, Comm. Pure Appl. Math. 12 (1959), 277-311.

[ 7 ] B. SMYth AND F. XAVIER, A sharp geometric estimate for the index of an umbilic on a smooth surface, Bull. London Math. Soc. 24 (1992), 176-180.

[ 8 ] B. SmYth And F. XAVIER, Real solvability of the equation $\partial_{\bar{z}}^{2} \omega=\rho g$ and the topology of isolated umbilics, J. Geom. Anal. 8 (1998), 655-671.

[ 9 ] C. J. Titus, A proof of a conjecture of Loewner and of the conjecture of Carathéodory on umbilic points, Acta Math. 131 (1973), 43-77.

DEPARTMENT OF MATHEMATICS

TOKYO METROPOLITAN UNIVERSITY

1-1 Minami-OHSAWA, HaChIOJI-SHI

TOKYO 192-0397

JAPAN

E-mail address: naoya@comp.metro-u.ac.jp 